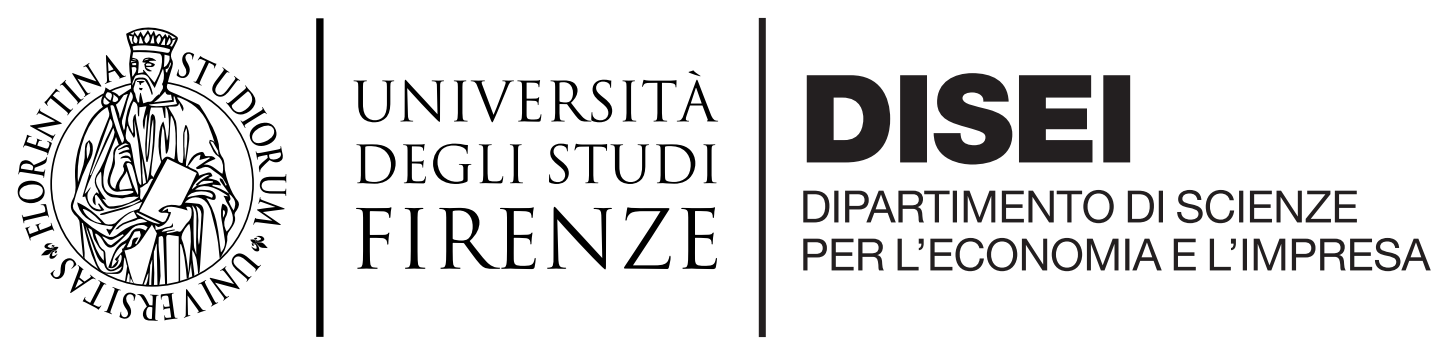

WORKING PAPERS - ECONOMICS

\title{
On the optimal entry fee and reserve price for auctions with selective entry: A comment on Gentry, Li, Lu (2017)
}

\author{
Nicola Doni And Domenico Menicucci
}

Working PAPER N. 18/2019 


\title{
On the optimal entry fee and reserve price for auctions with selective entry: A comment on Gentry, Li, Lu (2017)
}

\author{
Nicola Doni \\ (nicola.doni@unifi.it) \\ Dipartimento di Scienze per l'Economia e l'Impresa \\ Università degli Studi di Firenze, Via delle Pandette 32, I-50127 Firenze (FI), Italy \\ AND

\section{Domenico Menicucci*} \\ (domenico.menicucci@unifi.it) \\ Dipartimento di Scienze per l'Economia e l'Impresa \\ Università degli Studi di Firenze, Via delle Pandette 32, I-50127 Firenze (FI), Italy
}

May 31, 2019

\begin{abstract}
Gentry, Li, Lu (2017) (GLL henceforth) study an auction model with endogenous entry in which, before the entry decision, each bidder observes a private signal; a higher signal implies a better distribution for the bidder's valuation. GLL claim that the optimal reserve price is greater than the seller's value for the object on sale and that the optimal entry fee is positive. We prove that these claims are incorrect: The seller may want to subsidize entry to stimulate competition in the auction (through a negative entry fee or through a reserve price below the seller's value), or to provide appropriate entry incentives if a suitable reserve price is effective at maximizing total surplus and at extracting bidders' rents. We provide conditions under which the claims in GLL hold true.
\end{abstract}

JEL Classification: D44, D82.

Key words: Auctions; Endogenous Entry; Reserve price; Entry fee. 


\section{Introduction}

This note is about the optimal reserve price and entry fee for an auction with endogenous and costly entry. In a recent paper, Gentry, Li, Lu (2017) (GLL henceforth) assume that each (potential) bidder first observes a signal that reveals her value distribution, then decides whether to enter. Entry requires to incur an entry cost but allows a bidder to learn the own value and to bid in the auction. A higher signal is associated to a better distribution in the sense of first order stochastic dominance; this is consistent with various settings for which the information the signal conveys is very different. For instance, at an extreme is the model of Levin and Smith (1994), in which the value distribution is constant with respect to the signal; at another extreme is the setting of Menezes and Monteiro (2000), in which the bidder's value coincides with the bidder's signal.

For this model, GLL prove a Revenue Equivalence Theorem that covers a wide set of standard auction mechanisms and prove that the total surplus is maximized when the reserve price is equal to the seller's value $v_{0}$ and the entry fee is zero (in the following, sometimes we use $r, e$ to indicate the value of the reserve price and the value of the entry free, respectively). Furthermore, GLL claim that the revenue maximizing reserve price is strictly greater than $v_{0}$ and the revenue maximizing entry fee is strictly positive; the former (the latter) indication covers also the case in which $e$ is exogenously fixed at zero ( $r$ is exogenously fixed at $v_{0}$ ), the socially efficient level. Therefore, revenue maximization is always socially inefficient in the sense of inducing too rare entry and too little sales.

In this paper we show that these claims are incorrect, that is sometimes it is optimal for the seller to give an entry subsidy to bidders $(e<0)$ or to set $r$ below $v_{0}$, for instance to induce more than efficient entry and stimulate competition in the auction. We explain why the proofs in GLL of the above claims are incorrect and we provide (restrictive) conditions under which the claims in GLL hold true.

In detail, GLL determine an equilibrium in which each bidder enters if and only if her signal exceeds a threshold $s$, and claim that the bidders' ex ante expected rents are decreasing in $s$. However, we find that such monotonicity may not hold. We emphasize the presence of a trade-off: the higher the threshold, the lower the probability to enter (this contributes to reduce the expected rent), but the lower the intensity of competition faced by active bidders (this contributes to increase the expected rent). In some cases, this second effect dominates the first effect and makes rents (locally) increasing in $s$. Then, when $r$ is exogenously fixed at $v_{0}$, a small negative $e$ may be optimal because it has a tiny effect on total surplus (as the surplus maximizing entry fee is zero) but subsidizes bidders' entry and reduces $s$, thus increasing the revenue via a reduction in bidders' rents. When $e$ is fixed at zero, a similar mechanism applies as $r$ smaller than $v_{0}$ facilitates entry. Even though it induces too much entry and bidders buy the object too often with respect to the social optimum, in some cases $r<v_{0}$ increases revenue by reducing rents.

When the seller can choose both $r$ and $e$ freely, $r$ is set to maximize the revenue for a 
given $s$, and $e$ is used to achieve the desired $s$; thus $r$ does not affect entry. We show that the optimal $r$ may not be greater than $v_{0}$ if $v_{0}=0$, and that the optimal $e$ may be negative. The first result arises because, for a given $s$, the bidders' rents are approximately constant with respect to $r$ when $r$ is close to 0 . About the second result, as we mentioned above a negative $e$ may help to decrease bidders' rents, but also a very different reason may apply: for some information structures there exists an $r$ that (approximately) maximizes total surplus and extracts all rents. Then, by participating in the auction, bidders earn a payoff that is nearly zero and does not cover the entry cost. Then a negative entry fee is needed in order for some entry to occur.

In order to gain a perspective on these results, it is useful to recall that in the model of Levin and Smith (1994) (LS in the following), the optimal pair $r, e$ is $v_{0}, 0$. GLL claim that any devation from LS (within GLL's framework) leads a revenue maximizing seller to set $r>v_{0}, e>0$. We show that this is not the case, and in fact we prove that even very small deviations from LS yield ambiguous results about the optimal $e$, unless some further assumptions are imposed. Thus, the precise details of the information setting are important for a revenue maximizing seller, even from a qualitative point of view.

\section{The model}

A (male) seller auctions off an object and faces $N \geq 2$ potential (female) bidders; the seller and the bidders are risk neutral. At a first stage, each bidder $i$ privately observes a signal $s_{i} \in[0,1]$ which affects the distribution of her private valuation $v_{i}$ for the object on sale. Precisely, the support for $v_{i}$ is $[0, \bar{v}]$ with $\bar{v}>0$, and $F\left(\cdot \mid s_{i}\right)$ is the c.d.f. of $v_{i}$ given $s_{i}$ (with continuous density $f\left(\cdot \mid s_{i}\right)$ ) such that $s_{i}^{\prime}>s_{i}$ implies $F\left(v_{i} \mid s_{i}^{\prime}\right) \leq F\left(v_{i} \mid s_{i}\right)$ for each $v_{i} \in[0, \bar{v}]$, with strict inequality for at least one $v_{i}$. The bivariate random variables $\left(v_{i}, s_{i}\right),\left(v_{j}, s_{j}\right)$ are stochastically independent for each $i \neq j$, and each $s_{i}$ is uniformly distributed over $[0,1]$.

After observing the own signal, each bidder decides whether to enter the auction or not. Entry requires a bidder to incur a cost $c>0$ and the bidders' entry decisions are simultaneous. In a second stage each bidder that entered learns the own valuation and submits a bid in the auction. To fix the ideas, we can think that the auction is a second price auction, but GLL establish a Revenue Equivalence Theorem covering a class of auctions that extends well beyond the second price auction.

The seller has a value $v_{0} \in[0, \bar{v})$ for the object on sale and can charge an entry fee $e$ to each entrant; the entry fee is actually a subsidy if $e<0$. The seller can also impose a reserve price $r \geq 0$ in the auction. In the following, given a function $\Phi$ of two variables, we use $\Phi_{k}$ to denote the partial derivative of $\Phi$ with respect to its $k$-th variable, for $k=1,2$.

GLL identify a Bayes-Nash Equilibrium characterized by a signal value $s \in[0,1]$ which is the entry threshold: bidder $i$ enters if and only if $s_{i} \geq s$, for $i=1, \ldots, n$. To this purpose, 
it is useful to define

$$
F_{w}^{*}(v, s)=s+\int_{s}^{1} F(v \mid \sigma) d \sigma
$$

which is the probability that a given bidder does not enter, or enters and has a value smaller than $v$. Notice that $F_{w}^{*}$ is increasing with respect to $s$ as $F_{w 2}^{*}(v, s)=1-F(v \mid s) \geq 0$.

If bidder $i$ enters and discovers to have value $v_{i}$, then $F_{w}^{*}\left(v_{i}, s\right)$ is her probability to beat another given bidder; $\left(F_{w}^{*}\left(v_{i}, s\right)\right)^{N-1}$ is her probability to beat all other bidders. By Lemma 1 in Myerson (1981), the bidder's payoff is the integral of her probability to win the object, over the interval of values smaller than $v_{i}$; hence it is 0 if $v_{i} \leq r$, is $\int_{r}^{v_{i}}\left(F_{w}^{*}(v, s)\right)^{N-1} d v$ if $v_{i}>r$. At the entry stage, the payoff from entering for a bidder with signal $s_{i}$ is $\int_{r}^{\bar{v}}\left(\int_{r}^{v_{i}}\left(F_{w}^{*}(v, s)\right)^{N-1} d v\right) f\left(v_{i} \mid s_{i}\right) d v_{i}$, or $\int_{r}^{\bar{v}}\left(1-F\left(v \mid s_{i}\right)\right)\left(F_{w}^{*}(v, s)\right)^{N-1} d v$. The entry threshold $s$ is determined by the payoff of a bidder with signal $s_{i}=s$ compared with the total entry cost $c+e$. Therefore, $s=0$ if $\int_{r}^{\bar{v}}(1-F(v \mid 0))\left(F_{w}^{*}(v, 0)\right)^{N-1} d v \geq c+e$, $s=1$ if $\int_{r}^{\bar{v}}(1-F(v \mid 1)) d v \leq c+e$ (notice that $\left(F_{w}^{*}(v, 1)\right)^{N-1}=1$ for each $v$ ), otherwise $s$ is in the interval $(0,1)$ and bidder $i$ with signal $s_{i}=s$ is indifferent between entering and staying out. Hence $s$ is a solution of the following equation in $z$ :

$$
\int_{r}^{\bar{v}}(1-F(v \mid z))\left(F_{w}^{*}(v, z)\right)^{N-1} d v=c+e
$$

In fact, $s$ is determined uniquely as the left hand side in (1) is increasing in $z$. Notice that, for fixed $r, c$, for any given entry threshold $s$, the seller can induce that entry threshold by choosing $e$ that satisfies (1) when $z=s$. This allows to consider $s$ (rather than $e$ ) as a seller's policy instrument, with the interpretation that selecting a certain $s$ means that the seller picks the $e$ that induces the chosen $s$.

In order to determine the seller's revenue we derive the total surplus and the bidders' rents. The former is

$$
T S(r, s)=v_{0}\left(F_{w}^{*}(r, s)\right)^{N}+\int_{r}^{\bar{v}} v d\left(F_{w}^{*}(v, s)\right)^{N}-N(1-s) c
$$

For a bidder with signal $s_{i} \geq s$, the net rent from entering is $\int_{r}^{\bar{v}}\left(1-F\left(v \mid s_{i}\right)\right)\left(F_{w}^{*}(v, s)\right)^{N-1} d v-$ $c-e$, which reduces to $\int_{r}^{\bar{v}}\left(F_{w}^{*}(v, s)\right)^{N-1}\left(F(v \mid s)-F\left(v \mid s_{i}\right)\right) d v$ after using (1). Therefore a bidder's rent before observing the signal is

$$
\Pi(r, s)=\int_{r}^{\bar{v}}\left(F_{w}^{*}(v, s)\right)^{N-1}\left(\int_{s}^{1}(F(v \mid s)-F(v \mid \sigma)) d \sigma\right) d v
$$

Notice that $F(v \mid s) \geq F(v \mid \sigma)$ for each $\sigma>s$ implies $\Pi(r, s) \geq 0$ for each $r, s$. The revenue is the difference between the total surplus and the total bidders' rents, hence

$$
R(r, s)=T S(r, s)-N \Pi(r, s)
$$


In the following we are interested in maximizing $R$ with respect to $r$ and/or $s$, and we assume that

$$
\int_{v_{0}}^{\bar{v}}(1-F(v \mid 0))\left(F_{w}^{*}(v, 0)\right)^{N-1} d v<c<\int_{v_{0}}^{\bar{v}}(1-F(v \mid 1)) d v
$$

The second inequality in (5) rules out the uninteresting case in which it is optimal for the seller to induce no entry $(s=1)$, as that maximizes total surplus if $\int_{v_{0}}^{\bar{v}}(1-F(v \mid 1)) d v \leq$ $c$ and makes bidders' rents equal to 0 . About the first inequality in (5), if it is violated and $c<\int_{v_{0}}^{\bar{v}}(1-F(v \mid 0))\left(F_{w}^{*}(v, 0)\right)^{N-1} d v$ then $s=0$ when $(r, e)=\left(v_{0}, 0\right)$, that is each bidder $i$ enters for each $s_{i} \in[0,1]$. This makes it profitable for the seller to increase $e$ (for instance) at least up to the point where (1) holds given $r=v_{0}, z=0$. For the sake of brevity we focus on the case in which (5) holds, which implies that the entry threshold $s$ is in $(0,1)$ when $(r, e)=\left(v_{0}, 0\right)$ and any change in $r(e)$ away from $v_{0}$ (from 0 ) affects $s$.

An important result in GLL refers to the maximization of $T S$, for which it is useful to define $s_{0}$ as follows:

$$
s_{0} \text { is the solution of }(1) \text { when }(r, e)=\left(v_{0}, 0\right)
$$

GLL prove that $s_{0}\left(v_{0}\right)$ is the socially efficient value of $s$ (of $r$ ).

Lemma 1 (from GLL) The total surplus $T S$ is maximized at $r=v_{0}, s=s_{0}$.

As we show in next section, Lemma 1 is linked to the following key proposition in GLL.

Proposition 5 in GLL (Optimal entry fee and reserve) The optimal entry fee $e^{*}$ must be nonnegative and the optimal reserve $r^{*}$ must weakly exceed $v_{0}$. Furthermore, if entry is strictly selective in the sense that $s^{\prime}>s$ implies $F\left(v \mid s^{\prime}\right)<F(v \mid s)$ for some $v \in\left[v_{0}, \bar{v}\right]$, then the following statements hold:

(i) If the seller may set both $e$ and $r$ freely, then $e^{*}>0$ and $r^{*}>v_{0}$;

(ii) If the reserve is constrained efficient $\left(r=v_{0}\right)$, then the constrained optimum $e^{*}>0$;

(iii) If the entry fee is constrained zero $(e=0)$, then the constrained optimum $r^{*}>v_{0}$.

We prove in next section that this proposition is incorrect. To this purpose, we consider the following specific setting: For each bidder $i, F\left(v_{i} \mid s_{i}\right)=s_{i} G\left(v_{i}\right)+\left(1-s_{i}\right) H\left(v_{i}\right)$ with $G$, $H$ c.d.f. such that $G\left(v_{i}\right)<H\left(v_{i}\right)$ for each $v_{i} \in(0, \bar{v})$; moreover, $G(H)$ has a continuous density $g(h)$ which is positive at least in $(0, \bar{v})$. Hence, the c.d.f. for bidder $i$ 's valuation given $s_{i}$ is a mixture of a strong c.d.f. $(G)$ and a weak c.d.f. $(H)$ in which $s_{i}$ is the weight of the strong c.d.f. In next section we also give sufficient conditions for the claims (i-iii) in Proposition 5 in GLL to hold in this context. 


\section{On the optimal entry fee, reserve price}

In this section we show that in some settings it is revenue maximizing to set $e<0$ or $r<v_{0}$. To this purpose, it is useful to start with an analysis of the effect the entry threshold has on bidders' rents.

\subsection{The effect of $s$ on bidders' rents}

An important intermediate claim in GLL concerns the monotonicity of $\Pi$ in (3) with respect to $s$. It may be intuitive that bidders' rents decrease as the entry threshold increases, because that reduces the set of bidders that enter and there are fewer opportunities for bidders to earn a positive payoff by participating in the auction. However, it is important to recall that for a bidder that enters and turns out to have value $v_{i}>r$, the payoff from playing the auction is $\int_{r}^{v_{i}}\left(F_{w}^{*}(v, s)\right)^{N-1} d v$ and $F_{w}^{*}$ is increasing in $s$. This effect, which reduces the intensity of competition in the auction, goes in the opposite direction with respect to the effect above and in some cases dominates it. Indeed, although Lemma 4(iii) in GLL claims that $\Pi$ is decreasing with respect to $s$ (i.e., $\Pi_{2}(r, s) \leq 0$ for each $r, s$ ), we prove that this is not always the case. ${ }^{1}$

Lemma 2a The partial derivative of $\Pi$ with respect to $s$ is

$$
\Pi_{2}(r, s)=\frac{(N+1)(1-s)}{2} \int_{r}^{\bar{v}} K(v, s) d v
$$

with

$K(v, s)=\left(F_{w}^{*}(v, s)\right)^{N-2}\left(\frac{N-1}{N+1}-s-(1-s) G(v)-\frac{N}{N+1}(1-s)^{2}(H(v)-G(v))\right)(H(v)-G(v))$

It is immediate that $\Pi_{2}(r, s) \leq 0$ if $s \geq \frac{N-1}{N+1}$, or if (a weaker condition) $\frac{\frac{N-1}{N+1}-s}{1-s}-G(r) \leq$ 0 ; otherwise it is possible that $\Pi_{2}(r, s)>0$. Next lemma provides first a sufficient condition for $\Pi_{2}(r, s)<0$ for each $r, s$, then a sufficient condition for $\Pi_{2}(r, s)>0$ for some $r, s$. To this purpose, we pick $v^{G}$ such that $G\left(v^{G}\right)=\frac{N-1}{N+1}$ and, given $s^{\prime}<\frac{N-1}{N+1}$, we pick $v^{H}$ such that $H\left(v^{H}\right)=\left(\frac{N-1}{N+1}-s^{\prime}\right) /\left(1-s^{\prime}\right)$.

Lemma $2 \mathbf{b}(i)$ Given any $G$, suppose that $H(v)$ is very close to $G(v)$ for each $v \in\left(0, v^{G}\right]$. Then $\Pi_{2}(r, s)<0$ for each $r \in[0, \bar{v}), s \in[0,1)$.

(ii) Given any $H$ and any $s^{\prime}<\frac{N-1}{N+1}$, suppose that $G(v)$ is very close to $H(v)$ for each $v \in\left[v^{H}, \bar{v}\right)$. Then $\Pi_{2}(r, s)>0$ if $r<v^{H}$ and $s<s^{\prime}$.

\footnotetext{
${ }^{1}$ The proof of Lemma 4(iii) in GLL is incorrect because of a mistake in taking the partial derivative with respect to $s$ of $\left(F_{w}^{*}(v, s)\right)^{N-1}$ in (3).
} 
Lemma $2 \mathrm{~b}$ establishes that $\Pi_{2}(r, s)<0$ if $G, H$ almost coincide for small valuations, but $\Pi_{2}(r, s)>0$ holds if $r, s$ are both not too large and $G, H$ are very similar for large valuations. In next subsections we examine examples such that $\Pi_{2}(r, s)>0$, and show that this generates results which contrast with Proposition 5 in GLL. Before that, in order to gain further insights on the sign of $\Pi_{2}(r, s)$ we examine below two particular cases.

The case of degenerate $G, H$ Let $\hat{G}(\hat{H})$ denote the c.d.f. such that $\hat{G}(v)=0$ if $v<\bar{v}, \hat{G}(\bar{v})=1$, and $\hat{H}(v)=1$ for each $v \in[0, \bar{v}] .{ }^{2}$ Hence, $\hat{G}$ attaches probability 1 to $v=\bar{v}, \hat{H}$ attaches probability 1 to $v=0$. If $G=\hat{G}, H=\hat{H}$, then for each bidder $i$ the valuation is either 0 or $\bar{v}$ and $s_{i}$ is the probability of $v_{i}=\bar{v}$. From Lemma 2a we obtain $\Pi_{2}(r, s)=\frac{1-s}{2}\left(\frac{1}{2}+\frac{1}{2} s^{2}\right)^{N-2}(\bar{v}-r)\left(-N s^{2}+(N-1) s-1\right)$, hence $\Pi_{2}(r, s)<0$ for each $r, s$ if $N \leq 5$, but for $N \geq 6$ there exists an interval $S_{N} \subset(0,1)$ of values of $s$ which is centered in $\frac{N-1}{2 N}$ and such that $\Pi_{2}(r, s)>0$ for each $s \in S_{N}$; the extremes of $S_{N}$ tend to 0 and to 1, respectively, as $N$ grows to infinity.

Lemma 2c If $G, H$ are close to $\hat{G}, \hat{H}$, then $\Pi_{2}(r, s)<0$ for each $r, s$ if $N \leq 5$; when $N \geq 6, \Pi_{2}(r, s)$ is negative if $s$ is close to 0 or to 1 .

The case of $H$ close to $G$ This case is in a sense opposite to the previous one since we assume that $H$ is very similar to $G$. Precisely, given any $G$ with density $g$ such that $g(v)>0$ for each $v \in[0, \bar{v}]$, we consider a function $D:[0, \bar{v}] \rightarrow \mathbb{R}$ and $H$ such that

$$
\begin{gathered}
D(0)=D(\bar{v})=0, D(v)>0 \text { for each } v \in(0, \bar{v}), D \text { is continuously differentiable in }[0, \bar{v}] \\
H(v)=G(v)+\varepsilon D(v) \text { for each } v \in[0, \bar{v}], \text { with } \varepsilon \geq 0 \text { close to } 0
\end{gathered}
$$

If $\varepsilon=0$, then $H$ in (8) coincides with $G$. Hence $F$ is constant with respect to $s$ and $\Pi(r, s)=0$ for each $r, s$, thus $\Pi_{2}(r, s)=0$. If $\varepsilon>0,{ }^{3}$ then $H(v)>G(v)$ for each $v \in(0, \bar{v})$ and the effect on $\Pi_{2}(r, s)$ of a small increase in $\varepsilon$ above 0 is determined by

$$
\left.\frac{d \Pi_{2}(r, s)}{d \varepsilon}\right|_{\varepsilon=0}=\frac{(N+1)(1-s)}{2} \int_{r}^{\bar{v}}(s+(1-s) G(v))^{N-2}\left(\frac{N-1}{N+1}-s-(1-s) G(v)\right) D(v) d v
$$

It is immediate that for each given $D,(9)$ is negative if $G$ is a very weak c.d.f. (i.e., if $G(v)>\frac{N-1}{N+1}$ even for small $v$ ); (9) is positive if $s<\frac{N-1}{N+1}$ and $G$ is a very strong c.d.f. (i.e., $G$ is close to 0 for a wide subset of $(0, \bar{v}))$. Formally, recall from above that $v^{G}$ satisfies $G\left(v^{G}\right)=\frac{N-1}{N+1}$ and let $v_{s}$ satisfy $G\left(v_{s}\right)=\left(\frac{N-1}{N+1}-s\right) /(1-s)$. Then

\footnotetext{
${ }^{2}$ These c.d.f.s violate our assumption that $G(H)$ has a density, but the arguments here apply even if $G, H$ have continuous densities and $G(H)$ attaches a high probability to values close to $\bar{v}$ (close to 0 ).

${ }^{3}$ Notice that $H$ is a c.d.f. if and only if $h(v) \geq 0$ for each $v \in[0, \bar{v}]$. This condition is satisfied if $\varepsilon \leq \tilde{\varepsilon} \equiv \min _{v \in[0, v]} g(v) /\left(-\min _{v \in[0, v]} D^{\prime}(v)\right)$, and $\tilde{\varepsilon}>0$ in view of our assumptions on $G$ and in (8). Therefore we consider $\varepsilon \in[0, \tilde{\varepsilon}]$.
} 
Lemma 2d Suppose that $H$ is close to $G$ in the sense that (8) holds. Then

(i) $\Pi_{2}(r, s)<0$ if $v^{G}$ is sufficiently close to 0 ;

(ii) $\Pi_{2}(r, s)>0$ if $s<\frac{N-1}{N+1}$ and $v_{s}$ is sufficiently close to $\bar{v}$.

When $N=2$, we can determine a necessary and sufficient condition for (9) to be negative for each $r, s$. Given $N=2$, the integral in (9) is equal to $\int_{r}^{\bar{v}}\left(\frac{1}{3}-s-(1-\right.$ $s) G(v)) D(v) d v$, a linearly decreasing function of $s$. Hence (9) is negative for each $r, s$ if and only if $\int_{0}^{\bar{v}}\left(\frac{1}{3}-G(v)\right) D(v) d v<0 .^{4}$

\subsection{On the optimal $e$ when $r=v_{0}$ (Proposition 5(ii) in GLL)}

When $r$ is fixed at $v_{0}$, Lemma 1 implies that $s=s_{0}$ maximizes the total surplus $T S\left(v_{0}, s\right)$. If $\Pi_{2}\left(v_{0}, s\right) \leq 0$ for each $s$, then $-N \Pi\left(v_{0}, s\right)$ is increasing in $s$ and since $R\left(v_{0}, s\right)=$ $T S\left(v_{0}, s\right)-N \Pi\left(v_{0}, s\right)$, it follows that the value of $s$ that maximizes $R\left(v_{0}, s\right)$ is not smaller than $s_{0}$. Actually, it is greater than $s_{0}$ if $\Pi_{2}\left(v_{0}, s_{0}\right)<0$ as then $T S_{2}\left(v_{0}, s_{0}\right)=0$ implies $R_{2}\left(v_{0}, s_{0}\right)>0$; hence a positive $e$ is optimal. This is the argument in the proof of Proposition 5(ii) in GLL and it holds as long as $\Pi_{2}\left(v_{0}, s\right) \leq 0$ for each $s$, for instance if Lemma $2 \mathrm{~b}(\mathrm{i})$, or $2 \mathrm{c}$, or $2 \mathrm{~d}(\mathrm{i})$ applies.

However, in Subsection 3.1 we have remarked that $\Pi_{2}(r, s)>0$ in some cases. ${ }^{5}$ Next example exhibits a setting such that $\Pi_{2}\left(v_{0}, s_{0}\right)>0$ and the optimal $e$ is negative.

Example 1 Suppose that $N=2,[0, \bar{v}]=[0,1], v_{0}=0.25, G(v)=v^{10}, H(v)=v^{6}$, and $c=\int_{v_{0}}^{1}(1-F(v \mid 0.02))\left(F_{w}^{*}(v, 0.02)\right) d v=0.060143$, so that $s_{0}=0.02$. Then $\Pi_{2}\left(v_{0}, s_{0}\right)=$ 0.001293 and $R\left(v_{0}, s\right)$, (1) reduce, respectively, to

$$
\begin{gathered}
R\left(v_{0}, s\right)=-0.005171 s^{4}-0.060785 s^{3}-0.506298 s^{2}+0.01774 s+0.804514 \\
0.003448 z^{3}+0.05076 z^{2}+0.555878 z+0.049005=0.060143+e
\end{gathered}
$$

The revenue is maximized at $s=0.017464$, which is smaller than $s_{0}$ and therefore the optimal $e$ is negative. Indeed, the optimal $e$ satisfies (10) with $z=0.017464$, that is it is equal to $-0.001415<0$.

In Example 1, subsidizing entry through a (small) negative entry fee facilitates entry, that is reduces $s$ below $s_{0}$, therefore too much entry occurs from a social point of view. But this is profitable for the seller because the reduction in total surplus is tiny (as $T S_{2}\left(v_{0}, s_{0}\right)=0$ ) and the reduction in $s$ reduces bidders' rents as the more intense competition among active bidders dominates their more likely entry. Therefore the revenue increases, which establishes the following proposition:

\footnotetext{
${ }^{4}$ When $N=2$, a sufficient condition for $\Pi_{2}(r, s) \leq 0$ for each $r, s$ is that there exists $\varepsilon$ close to zero and $\alpha \in(0,1)$ such that $H(v)>1-\varepsilon$ for each $v \geq \varepsilon$ and $H(v)-G(v)>\alpha$ in an interval included in $(\varepsilon, \bar{v})$ with lenght at least $\frac{4 \varepsilon}{3 \alpha}$.

${ }^{5}$ We could rely on Lemmas $2 \mathrm{~b}(\mathrm{ii}), 2 \mathrm{~d}(\mathrm{ii})$ to identify a setting such that $\Pi_{2}(r, s)>0$. However, in Example 1 we select a simpler setting than those covered by the above lemmas.
} 
Proposition 1 If the reserve price is constrained at $v_{0}$, then the optimal entry fee may be negative.

\subsection{On the optimal $r$ when $e=0$ (Proposition 5(iii) in GLL)}

When $e$ is fixed at 0 , the entry threshold given $r$ is obtained by solving (1) and is denoted $\tilde{s}(r) .{ }^{6}$ Notice that (6) implies $\tilde{s}\left(v_{0}\right)=s_{0}$, and $\tilde{s}^{\prime}(r)$ is positive by implicit differentiation.

Given that $e=0$, it is useful to define total surplus, rents, revenue as a function of $r$ only: $\widetilde{T S}(r)=T S(r, \tilde{s}(r)), \tilde{\Pi}(r)=\Pi(r, \tilde{s}(r)), \tilde{R}(r)=R(r, \tilde{s}(r))$. Then $\tilde{s}\left(v_{0}\right)=s_{0}$ and Lemma 1 imply that $\widetilde{T S}$ is maximized at $r=v_{0}$, hence $\widetilde{T S}^{\prime}\left(v_{0}\right)=0$. Moreover, if $\Pi_{2}(r, s) \leq 0$ for each $r, s$ then $\tilde{\Pi}$ is decreasing as $\tilde{\Pi}^{\prime}(r)=\Pi_{1}(r, \tilde{s}(r))+\Pi_{2}(r, \tilde{s}(r)) \tilde{s}^{\prime}(r)$ is negative or zero since $\Pi_{1}(r, s) \leq 0, \tilde{s}^{\prime}(r)>0$. Therefore, the value of $r$ that maximizes $\tilde{R}$ is not smaller than $v_{0}$; actually, it is greater than $v_{0}$ if $\Pi_{2}\left(v_{0}, s_{0}\right)<0$ since then $\widetilde{T S}\left(v_{0}\right)=0$ implies $\tilde{R}^{\prime}\left(v_{0}\right)>0$. This is the argument in the proof of Proposition 5(iii) in GLL and holds as long as $\Pi_{2}(r, s) \leq 0$ for each $r, s$, for instance if Lemma $2 \mathrm{~b}(\mathrm{i})$, or $2 \mathrm{c}$, or $2 \mathrm{~d}(\mathrm{i})$ applies.

However, in Example 1 above we have seen that $\Pi_{2}\left(v_{0}, s_{0}\right)>0$ and this makes $\tilde{\Pi}$ increasing for $r$ close to $v_{0} \cdot{ }^{7}$ Hence $\tilde{R}$ is decreasing for $r$ close to $v_{0}$ and we find that the optimal $r$ is smaller than $v_{0}$.

Example 2 Consider the same setting as in Example 1. ${ }^{8}$ Then $\Pi_{1}\left(v_{0}, s_{0}\right)=-2.349 \times 10^{-6}$, $\Pi_{2}\left(v_{0}, s_{0}\right)=0.001293$, and from $\tilde{s}^{\prime}(r)=\frac{(1-F(r \mid \tilde{s}(r))) F_{w}^{*}(r, \tilde{s}(r))}{\int_{r}^{1}\left((H(v)-G(v)) F_{w}^{*}(v, \tilde{s}(r))+(1-F(v \mid \tilde{s}(r)))^{2}\right) d v}>0$ we obtain $\tilde{s}^{\prime}\left(v_{0}\right)=0.03605$. Thus $\tilde{\Pi}^{\prime}\left(v_{0}\right)=4.425 \times 10^{-5}>0$ and $\tilde{\Pi}$ is increasing for $r$ around $v_{0}$; therefore $\tilde{R}$ is decreasing for $r$ around $v_{0}$. This suggests that the optimal $r$ is smaller than $v_{0}=0.25$, and indeed numerical analysis shows that the optimal $r$ is 0.170822 . The resulting entry threshold is $\tilde{s}(0.170822)=0.017508<s_{0}=0.02$.

The intuition for Example 2 in part coincides with that for Example 1. Reducing $r$ slightly below $v_{0}$ reduces $s$, and the net effect on $\Pi$ is determined by a positive direct effect of $r$ plus a negative indirect effect through $s$; the second effect dominates over the first, hence $\Pi$ is reduced. Too much entry and too many sales reduce total surplus, but since $\widetilde{T S}\left(v_{0}\right)=0$ this effect is negligible with respect to the rent reduction. Therefore the revenue increases, which establishes the following proposition:

Proposition 2 If the entry fee is constrained at 0 , then the optimal reserve price may be lower than $v_{0}$.

\footnotetext{
${ }^{6}$ In fact, $\tilde{s}(r)$ is obtained by solving (1) (given $e=0$ ) if $\int_{r}^{\bar{v}}(1-F(v \mid 0))\left(F_{w}^{*}(v, 0)\right)^{N-1} d v \leq c \leq$ $\int_{r}^{\bar{v}}(1-F(v \mid 1)) d v$. In case the first (second) former inequality is violated, then $\tilde{s}(r)=0(\tilde{s}(r)=1)$. Assumption (5) guarantees that there exists a neighborhood of $v_{0}$ such that $\tilde{s}(r)$ solves (1) for each $r$ in the neighborhood; this suffices for our point in this subsection.

${ }^{7}$ The effect of $\Pi_{2}\left(v_{0}, s_{0}\right)>0$ dominates over $\Pi_{1}\left(v_{0}, s_{0}\right)<0$ because $\Pi_{1}\left(v_{0}, s_{0}\right)$ is very close to zero.

${ }^{8}$ The neighborhood of $v_{0}=0.25$ mentioned in Footnote 6 is $[0,0.8681] ; \tilde{s}(r)=1$ if $r>0.8681$.
} 


\subsection{On the optimal $r, e$ (Proposition 5(i) in GLL)}

When there are no constraints on $r, e$, the seller can choose freely $r, s$ to maximize his revenue in (4); we use $r^{*}, s^{*}, e^{*}$ to denote the optimal $r, s, e$, respectively.

\subsubsection{If $v_{0}=0$, then $r^{*}$ may not be larger than $v_{0}$}

About $r^{*}$, GLL notice that for each $s, T S$ is maximized with respect to $r$ at $r=v_{0}$ and that $\Pi$ is decreasing with respect to $r$ as $\Pi_{1}(r, s)=-\left(F_{w}^{*}(r, s)\right)^{N-1} \int_{s}^{1}(F(r \mid s)-F(r \mid \sigma)) d \sigma \leq 0$; hence $r^{*} \geq v_{0}$. As we mentioned in the introduction, LS proves that if each bidder has no information about the own value before entry, then $r^{*}=v_{0}, e^{*}=0$. This occurs because bidder $i$ 's signal $s_{i}$ conveys no information about $i$ 's value if $F$ is constant with respect to $s_{i}$; then $\Pi(r, s)$ in $(3)$ is constantly zero and $r, e$ are used to maximize TS. Proposition $5(\mathrm{i})$ in GLL claims that, outside the setting in LS, $r^{*}$ is larger than $v_{0}$. This in fact holds true as long as $\Pi_{1}\left(v_{0}, s\right)<0$, but even when $F$ depends on $s_{i}$, this inequality is violated if $v_{0}=0$ because $F\left(0 \mid s_{i}\right)=0$ for each $s_{i}$. Then $\Pi_{1}(0, s)=0,{ }^{9}$ and a small increase in $r$ above 0 has a second order effect on $T S$ and $\Pi$, hence it is not necessarily profitable. Precisely, it is possible that $\Pi$ decreases with respect to $r$ more slowly than $T S$, which leads to $r^{*}=0$. Next proposition gives sufficient conditions for $r^{*}=0$, and for $r^{*}>0 .{ }^{10}$

Proposition 3 When $v_{0}=0$,

(i) the optimal reserve price is zero if

$$
H(v)-G(v) \leq v g(v)+v h(v) \quad \text { for each } v \in(0, \bar{v})
$$

(ii) the optimal reserve price is positive if $g(0)=0, g^{\prime}(0)=0, h^{\prime}(0)<0$.

A sufficient condition for (11) to hold is that $H(v)$ is close to $G(v)$ for each $v \in(0, \bar{v})$. This is linked to the result of LS: In our mixture model, $F$ is constant with respect to $s_{i}$ if and only if $G$ coincides with $H$; then (11) holds and $r^{*}=v_{0}=0$ consistently with LS. Proposition 3(i) extends this result to cases in which $G$ is different from $H$ but is close. Therefore $r^{*}=v_{0}$ still holds for settings close to LS if $v_{0}=0$. Finally, we notice that another sufficient condition for (11) if that $H$ is convex, as then $H(v) \leq v h(v)$ for each $v$.

\subsubsection{The optimal $e$ may be negative}

About $e^{*}$, following GLL we can use (2)-(4) to derive

$$
R_{2}(r, s)=\left(v_{0}-r\right) \frac{\partial\left(F_{w}^{*}(r, s)\right)^{N}}{\partial s}-N \int_{r}^{\bar{v}}\left(F_{w}^{*}(v, s)\right)^{N-1}[1-F(v \mid s)] d v+N c-N \Pi_{2}(r, s)
$$

\footnotetext{
${ }^{9}$ The effect of an increase in $r$ on $\Pi$ is determined by the effect on the gross rents of bidders with signal above $s$, minus the effect on the gross rent of the bidder with signal equal to $s$. Both rents are decreasing in $r$, but when $v_{0}=0$ a small positive $r$ has a zero first order effect on the difference.

${ }^{10}$ Proposition 5(i) in GLL holds relative to $r^{*}$ if $v_{0} \in(0, \bar{v})$ and $F$ is strictly decreasing in the signal for each $v \in(0, \bar{v})$. For instance, this holds in our mixture model as $G(v)<H(v)$ for each $v \in(0, \bar{v})$.
} 
Then suppose that $r^{*}, s^{*}$ satisfies $R_{2}\left(r^{*}, s^{*}\right)=0,{ }^{11}$ and use (1) to obtain

$$
e^{*}=\frac{1}{N}\left(v_{0}-r^{*}\right) \frac{\partial\left(F_{w}^{*}\left(r^{*}, s^{*}\right)\right)^{N}}{\partial s}-\Pi_{2}\left(r^{*}, s^{*}\right)
$$

When $v_{0}=0$ and Proposition 3(i) applies, we have that $r^{*}=0$ and (12) reduces to $e^{*}=-\Pi_{2}\left(0, s^{*}\right)$. Then Lemmas $2 \mathrm{~b}$-d provide sufficient conditions for $e^{*}>0$, but relying on the logic of Subsection 3.2 we can identify examples such that $e^{*}<0$. For instance, Example 1 with $v_{0}=0$ is such that $H$ is convex, hence (11) is satisfied and $r^{*}=0$. Although $s_{0}=0.0138$, the optimal $s$ is 0.0117 , smaller than $s_{0}$, and $e^{*}=-0.0017<0$.

When $v_{0}>0$, we have that $r^{*}>v_{0}$ and $\frac{\partial\left(F_{w}^{*}\left(r^{*}, s^{*}\right)\right)^{N}}{\partial s}>0$. Therefore $\frac{1}{N}\left(v_{0}-\right.$ $\left.r^{*}\right) \frac{\partial\left(F_{w}^{*}\left(r^{*}, s^{*}\right)\right)^{N}}{\partial s}<0$ and since $\Pi_{2}\left(r,{ }^{*} s^{*}\right)$ may have either sign, further assumptions are needed to derive a conclusion about the sign of $e^{*}$. For instance, $\Pi_{2}\left(r,{ }^{*} s^{*}\right)$ needs to be sufficiently negative in order to for $e^{*}$ to be positive. ${ }^{12}$ In the following we examine the two particular cases we have introduced in Subsection 3.1.

The case of degenerate $G, H$ When $G=\hat{G}$ and $H=\hat{H}$, it is optimal for the seller to set $r$ slightly lower than $\bar{v}$ and $e$ negative. The reason is that for each bidder $i$ the valuation is either 0 or $\bar{v}$, hence each $r$ in $(0, \bar{v}]$ maximizes total surplus in the auction (the object is sold if and only if at least a bidder has value $\bar{v}$ ), but $r$ close to $\bar{v}$ also extracts almost all bidders' rents from playing the auction. As a consequence, no bidder can expect to recover the entry cost: even if the bidder turns out to have value $\bar{v}$ and wins the auction, she will pay at least $r$ for the object, which is close to $\bar{v}$. Therefore a negative $e$ is needed to induce some entry, and since the seller leaves virtually no rents to bidders, it is optimal to select $e$ in order to induce the socially efficient entry.

Proposition 4 If $G, H$ are close to $\hat{G}, \hat{H}$, then $r^{*}$ is close to $\bar{v}$ and $e^{*}<0$.

We remark that in this setting the argument of Subsection 3.2 for $e<0$ does not apply. Indeed, if $N=2$ (for instance) then $\Pi$ is monotonically decreasing with respect to $s$ by Lemma 2c. Actually, here a suitable $r$ is effective at extracting bidders rents (without hurting total surplus), hence there is no point in using a subsidy to induce a socially excessive entry in order to stimulate competition among bidders. Rather, the role of the entry subsidy is to cover almost entirely the entry cost, otherwise no entry occurs.

\footnotetext{
${ }^{11}$ This is not necessarily the case if $s^{*}=0$ as then $R_{2}\left(r^{*}, s^{*}\right)$ may be negative.

${ }^{12}$ The proof of Proposition 5(i) in GLL considers $\frac{\partial\left(F_{w}^{*}\left(r^{*}, s^{*}\right)\right)^{N}}{\partial s}$ as negative (whereas it is positive, as GLL note elsewhere in the paper), hence it considers $\frac{1}{N}\left(v_{0}-r^{*}\right) \frac{\partial\left(F_{w}^{*}\left(r^{*}, s^{*}\right)\right)^{N}}{\partial s}$ as positive since $v_{0}-r^{*}<0$, and finally considers $\Pi_{2}\left(r^{*}, s^{*}\right)$ as negative or zero (whereas it may be positive). Therefore it incorrectly concludes from (12) that $e^{*}>0$.
} 
The case of $H$ close to $G$ Suppose that $G, H$ satisfy (8). Hence $H$ coincides with $G$ if $\varepsilon=0$, and then $r^{*}=v_{0}, e^{*}=0$ by LS. We use $s_{0}$ to denote the resulting entry threshold from (1), which is also the socially optimal entry threshold when $\varepsilon=0$. We examine the effect of a small increase in $\varepsilon$ above 0 (which implies $H(v)>G(v)$ for each $v \in(0, \bar{v})$ ) on $r^{*}, s^{*}, e^{*}$. To this purpose, we define $L(v)$ as $s_{0}+\left(1-s_{0}\right) G(v) ; L(v)$ coincides with $F_{w}^{*}\left(v, s_{0}\right)$ if $H=G$.

Lemma 3 Suppose that $v_{0} \in(0, \bar{v})$ and that $H$ is close to $G$ in the sense described by (8). Let $r^{*}(\varepsilon), s^{*}(\varepsilon), e^{*}(\varepsilon)$ denote the optimal $r, s, e$ as a function of $\varepsilon$. Then

$$
\begin{aligned}
r^{* \prime}(0) & =\frac{\left(1-s_{0}\right) D\left(v_{0}\right)}{2 g\left(v_{0}\right)}, \quad s^{* \prime}(0)=\frac{\left(1-s_{0}\right)(N+1) \int_{v_{0}}^{\bar{v}} D(v)(L(v))^{N-2}\left(L(v)-\frac{N-1}{N+1}\right) d v}{(N-1) \int_{v_{0}}^{\bar{v}}(L(v))^{N-2}(1-G(v))^{2} d v} \\
e^{* \prime}(0) & =\frac{1-s_{0}}{2}\left((N+1) \int_{v_{0}}^{\bar{v}} D(v)(L(v))^{N-2}\left(L(v)-\frac{N-1}{N+1}\right) d v-D\left(v_{0}\right)\left(L\left(v_{0}\right)\right)^{N-1} \frac{1-G\left(v_{0}\right)}{g\left(v_{0}\right)}\right)
\end{aligned}
$$

Since $v_{0}>0$, from $r^{* \prime}(0)=\frac{\left(1-s_{0}\right) D\left(v_{0}\right)}{2 g\left(v_{0}\right)}$ we see that a small increase in $\varepsilon$ unambiguously increases $r^{*}$, consistently with Footnote 10. Conversely, $e^{* \prime}(0)$ may have either sign. In order for $e^{* \prime}(0)$ to be positive it suffices that $\int_{v_{0}}^{\bar{v}} D(v)(L(v))^{N-2}\left(L(v)-\frac{N-1}{N+1}\right) d v>0$ and $v_{0}$ is close to zero, as then $D\left(v_{0}\right)\left(L\left(v_{0}\right)\right)^{N-1} \frac{1-G\left(v_{0}\right)}{g\left(v_{0}\right)}$ is about zero. Next proposition relies on some sufficient conditions for $\int_{v_{0}}^{\bar{v}} D(v)(L(v))^{N-2}\left(L(v)-\frac{N-1}{N+1}\right) d v>0$.

Proposition 5 Suppose that $H$ is close to $G$ in the sense that (8) holds for a small $\varepsilon>0$, that $v_{0}$ is close to 0 and either $s_{0}>\frac{N-1}{N+1}$, or $v^{G}$ is close to 0 . Then $r^{*}>v_{0}, e^{*}>0$.

When Proposition 5 cannot be applied, typically $D$ plays a role in determining the sign of $e^{* \prime}(0)$. For instance, if $[0, \bar{v}]=[0,1], G$ is the uniform distribution, $D(v)=v(1-v)$, then $e^{\prime}(0)>0$ for each $s_{0} \in(0,1), v_{0} \in(0,1)$. But if we change $D(v)$ into $v(1-v)^{2}$, then $H$ puts less weight on low values than when $D(v)=v(1-v)$ and $e^{* \prime}(0)>0$ if $v_{0}<0.276$, $e^{* \prime}(0)<0$ if $v_{0}>\frac{1}{3}$; the sign of $e^{* \prime}(0)$ depends also on $s_{0}$ if $v_{0}$ is between 0.276 and $\frac{1}{3}$.

\section{Conclusions}

GLL examine an entry model under relatively weak assumptions on how significant each bidder's information is at the entry stage. Whereas some of their results hold for each particular specification of the model, we prove that this is not the case for their claims regarding the optimal entry fee and reserve price. More specifically, contradicting their conclusions, we show examples in which the seller may increase the revenue by favoring bidders' participation in the auction either with a negative entry fee, or with a reserve price lower than his value for the object. In general, the optimal auction may require a participation either lower or higher than the socially optimal level depending on the specific details of the context, and no broad conclusion is possible. 


\section{References}

[1] Gentry, M., Li, T., Lu, J., 2017. Auctions with selective entry. Games and Economic Behavior 105, 104-111.

[2] Levin, D., Smith, J.L., 1994. Equilibrium in auctions with entry. American Economic Review, 84, 585-599.

[3] Menezes, F.M., Monteiro, P.K., 2000. Auctions with endogenous participation. Review of Economic Design, 5, 71-89.

[4] Myerson, R.B., 1981. Optimal Auction Design. Mathematics of Operations Research $6,58-73$.

\section{Appendix}

\subsection{Proof of Lemma 2a}

From (3) we obtain

$$
\Pi_{2}(r, s)=\int_{r}^{\bar{v}}\left(\begin{array}{c}
(N-1)\left(F_{w}^{*}(v, s)\right)^{N-2}(1-F(v \mid s)) \int_{s}^{1}(F(v \mid s)-F(v \mid \sigma)) d \sigma \\
+\left(F_{w}^{*}(v, s)\right)^{N-1} \int_{s}^{1} F_{2}(v \mid s) d \sigma
\end{array}\right) d v
$$

Then $F(v \mid s)=s G(v)+(1-s) H(v)$ yields $\int_{s}^{1}(F(v \mid s)-F(v \mid \sigma)) d \sigma=\frac{1}{2}(1-s)^{2}(H(v)-$ $G(v))$ and $\int_{s}^{1} F_{2}(v \mid s) d \sigma=-(1-s)(H(v)-G(v))$. Hence

$\Pi_{2}(r, s)=(1-s) \int_{r}^{\bar{v}}\left(F_{w}^{*}(v, s)\right)^{N-2}\left(\frac{N-1}{2}(1-s)(1-F(v \mid s))-F_{w}^{*}(v, s)\right)(H(v)-G(v)) d v$

Since $F_{w}^{*}(v, s)=s+\frac{1-s}{2}(G(v)+H(v)+s G(v)-s H(v))$, simple manipulations lead to (7).

\subsection{Proof of Lemma $2 b$}

(i) For each $v>v^{G}, G(v)>\frac{N-1}{N+1}$ hence $\frac{N-1}{N+1}-s-(1-s) G(v)<0$ and $K(v, s)<0$. Taking $r$ into account, we see that if $v^{G} \leq r<\bar{v}$, then $K(v, s)<0$ for each $v \in(r, \bar{v})$; hence $\Pi_{2}(r, s)<0$. If $r<v^{G}$, then $K(v, s)<0$ for each $v \in\left(v^{G}, \bar{v}\right)$. For $v \in\left(r, v^{G}\right]$, $K(v, s)$ may be positive but is close to zero as $H(v)-G(v)$ is close to zero. Hence $\int_{r}^{\bar{v}} K(v, s) d v=\int_{r}^{v^{G}} K(v, s) d v+\int_{v^{G}}^{\bar{v}} K(v, s) d v<0$ and $\Pi_{2}(r, s)<0$.

(ii) We notice that $K(v, s)=\left(F_{w}^{*}(v, s)\right)^{N-2}(H(v)-G(v)) M(v, s)$ with $M(v, s)=\frac{N-1}{N+1}-$ $s-(1-s) G(v)-\frac{N}{N+1}(1-s)^{2}(H(v)-G(v))$, and consider $v \in\left(0, v^{H}\right), s<s^{\prime}$. If $G(v)$ were equal to $H(v)$, then $M(v, s)$ would be $\frac{N-1}{N+1}-s-(1-s) H(v)>\frac{N-1}{N+1}-s-(1-s) H\left(v^{H}\right)=$ 
$(1-s)\left(\frac{\frac{N-1}{N+1}-s}{1-s}-\frac{\frac{N-1}{N+1}-s^{\prime}}{1-s^{\prime}}\right)>0$. Moreover, $M(v, s)$ is decreasing in $G(v)$ hence $M(v, s)>0$ given $G(v)<H(v)$, thus $K(v, s)=\left(F_{w}^{*}(v, s)\right)^{N-2}(H(v)-G(v)) M(v, s)>0$ for each $v \in\left(0, v^{H}\right)$. Therefore, given $r<v^{H}, \int_{r}^{v^{H}} K(v, s) d v>0$ and $\int_{v^{H}}^{\bar{v}} K(v, s) d v$ may be negative but is close to zero as $H(v)-G(v)$ is close to zero in the interval $\left(v^{H}, \bar{v}\right)$. Thus $\int_{r}^{\bar{v}} K(v, s) d v=\int_{r}^{v^{H}} K(v, s) d v+\int_{v^{H}}^{\bar{v}} K(v, s) d v>0$ and $\Pi_{2}(r, s)>0$.

\subsection{Proof of Proposition 3}

When $v_{0}=0$, from (2)-(4) we obtain $R_{1}(r, s)=\frac{N(1-s)}{2}\left(F_{w}^{*}(r, s)\right)^{N-1} z(r, s)$ with $z(r, s)=$ $H(r)-r h(r)-G(r)-r g(r)+s(G(r)+r h(r)-H(r)-r g(r))$.

(i) We prove that $(11)$ implies $z(r, s) \leq 0$ for each $r, s$. First notice that $z(r, s)$ is linear in $s$, and the inequality $z(r, 0) \leq 0$ is equivalent to $H(r)-G(r) \leq r g(r)+r h(r)$, which is just (11). Moreover, $z(r, 1) \leq 0$ reduces to $-2 r g(r) \leq 0$, which holds for each $r$. Therefore (11) suffices for $z(r, s) \leq 0$ for each $r, s$.

(ii) It is immediate that $z(0, s)=0$. In the following we prove that $z_{1}(0, s)=0, z_{11}(0, s)>$ $0\left(z_{11}\right.$ is the second derivative of $z$, twice with respect to $\left.r\right)$ hence $z_{1}(r, s)>0$ for $r>0$ close to 0 and finally $z(r, s)>0$ for $r>0$ close to zero. Therefore a small positive reserve price is superior to $r=0$. In detail, $z_{1}(r, s)=-r h^{\prime}(r)-r g^{\prime}(r)+s r h^{\prime}(r)-s r g^{\prime}(r)-2 g(r)$ and $z_{1}(0, s)=-2 g(0)$ is zero as $g(0)=0$. Moreover, $z_{11}(r, s)=-h^{\prime}(r)-r h^{\prime \prime}(r)-3 g^{\prime}(r)-$ $r g^{\prime \prime}(r)+s\left(h^{\prime}(r)+r h^{\prime \prime}(r)-g^{\prime}(r)-r g^{\prime \prime}(r)\right)$ and $z_{11}(0, s)=-(3+s) g^{\prime}(0)-(1-s) h^{\prime}(0)$ is positive as $g^{\prime}(0)=0, h^{\prime}(0)<0$.

\subsection{Proof of Proposition 4}

From (2)-(4) and integration by parts we obtain

$$
\begin{aligned}
R(r, s)= & \left(v_{0}-r\right)\left(F_{w}^{*}(r, s)\right)^{N}+\bar{v}-\int_{r}^{\bar{v}}\left(F_{w}^{*}(v, s)\right)^{N} d v-N(1-s) c \\
& -N \int_{r}^{\bar{v}}\left(F_{w}^{*}(v, s)\right)^{N-1}\left(\int_{s}^{1}(F(v \mid s)-F(v \mid \sigma)) d \sigma\right) d v
\end{aligned}
$$

Since $F\left(v \mid s_{i}\right)=1-s_{i}, F_{w}^{*}(v, s)=\frac{1}{2}+\frac{1}{2} s^{2}$, the revenue boils down to $\bar{v}-\left(\bar{v}-v_{0}\right)\left(\frac{1}{2}+\frac{1}{2} s^{2}\right)^{N}-$ $N(1-s) c-N\left(\frac{1}{2}+\frac{1}{2} s^{2}\right)^{N-1} \frac{1}{2}(1-s)^{2}(\bar{v}-r)$. This is increasing in $r$, which suggests to set $r=\bar{v}$ or slightly smaller. The socially optimal $s$ maximizes $\bar{v}-\left(\bar{v}-v_{0}\right)\left(\frac{1}{2}+\frac{1}{2} s^{2}\right)^{N}-$ $N(1-s) c$, and denoting it with $s_{0}$ we conclude that the optimal $e$ is obtained from (1) with $z=s_{0}$. Hence it is equal to $s_{0}\left(\frac{1}{2}+\frac{1}{2} s_{0}^{2}\right)^{N-1}(\bar{v}-r)-c$, which is negative given $r$ about $\bar{v}$. 


\subsection{Proof of Lemma 3}

From $R(r, s)$ in the proof of Proposition 4 we obtain

$$
\begin{aligned}
& R_{1}(r, s)=\frac{N(1-s)}{2}\left(F_{w}^{*}(r, s)\right)^{N-1}\left(\left(v_{0}-r\right)(2 g(r)+(1-s) \varepsilon d(r))+(1-s) \varepsilon D(r)\right) \\
& R_{2}(r, s)=N\left(\left(v_{0}-r\right)\left(F_{w}^{*}(r, s)\right)^{N-1}[1-F(r \mid s)]-\int_{r}^{\bar{v}}\left(F_{w}^{*}(v, s)\right)^{N-1}[1-F(v \mid s)] d v+c-\Pi_{2}(r, s)\right)
\end{aligned}
$$

in which $d(v)=D^{\prime}(v)$. Therefore $r^{*}, s^{*}$ solve the system

$$
A(r, s, \varepsilon)=0, \quad B(r, s, \varepsilon)=0
$$

with $A(r, s, \varepsilon)=\left(v_{0}-r\right)(2 g(r)+(1-s) \varepsilon d(r))+(1-s) \varepsilon D(r)$ and $B(r, s, \varepsilon)=\left(v_{0}-\right.$ $r)\left(F_{w}^{*}(r, s)\right)^{N-1}[1-F(r \mid s)]-\int_{r}^{\bar{v}}\left(F_{w}^{*}(v, s)\right)^{N-1}[1-F(v \mid s)] d v+c-\Pi_{2}(r, s)$. Since $H(v)=$ $G(v)+\varepsilon D(v)$, it follows that $F_{w}^{*}(v, s)=s+(1-s) G(v)+\frac{(1-s)^{2}}{2} \varepsilon D(v), 1-F(v \mid s)=$ $1-G(v)-(1-s) \varepsilon D(v), \Pi_{2}(r, s)=\frac{(N+1)(1-s)}{2} \int_{r}^{\bar{v}}\left(s+(1-s) G(v)+\frac{(1-s)^{2}}{2} \varepsilon D(v)\right)^{N-2}\left(\left(\frac{N-1}{N+1}-\right.\right.$ $\left.s-(1-s) G(v)) \varepsilon D(v)-\frac{N}{N+1}(1-s)^{2} \varepsilon^{2} D^{2}(v)\right) d v$.

Now we apply the Implicit function theorem to the system $(13)$ at $(r, s, \varepsilon)=\left(v_{0}, s_{0}, 0\right)$ to derive $r^{* \prime}(0), s^{* \prime}(0), e^{* \prime}(0)$. We obtain $A_{1}\left(v_{0}, s_{0}, 0\right)=-2 g\left(v_{0}\right), A_{2}\left(v_{0}, s_{0}, 0\right)=0, A_{3}\left(v_{0}, s_{0}, 0\right)=$ $\left(1-s_{0}\right) D\left(v_{0}\right)$, from which $r^{* \prime}(0)$ in Lemma 3 follows. Moreover, $B_{1}\left(v_{0}, s_{0}, 0\right)=0$, $B_{2}\left(v_{0}, s_{0}, 0\right)=-(N-1) \int_{v_{0}}^{\bar{v}}(L(v))^{N-2}(1-G(v))^{2} d v, B_{3}\left(v_{0}, s_{0}, 0\right)=(N+1)\left(1-s_{0}\right) \int_{v_{0}}^{\bar{v}} D(v)$ $(L(v))^{N-2}\left(L(v)-\frac{N-1}{N+1}\right) d v$, hence $s^{* \prime}(0)$ in Lemma 3 is obtained.

Finally, $e=\int_{r}^{\bar{v}}(1-G(v)-(1-s) \varepsilon D(v))\left(s+(1-s) G(v)+\frac{(1-s)^{2}}{2} \varepsilon D(v)\right)^{N-1} d v-c$ from $(1)$, therefore $e^{* \prime}(0)=r^{* \prime}(0)\left(-\left(1-G\left(v_{0}\right)\right)\left(L\left(v_{0}\right)\right)^{N-1}\right)+s^{* \prime}(0)(N-1) \int_{v_{0}}^{\bar{v}}(L(v))^{N-2}(1-$ $G(v))^{2} d v-\frac{1}{2}(N+1)\left(1-s_{0}\right) \int_{v_{0}}^{\bar{v}} D(v)(L(v))^{N-2}\left(L(v)-\frac{N-1}{N+1}\right) d v$, which yields $e^{* \prime}(0)$ in Lemma 3 . 